\title{
Coding to reduce the interference to carrier ratio of OFDM signals
}

\author{
Besma Smida (D)
}

\begin{abstract}
In this paper, we propose a simple approach to reduce the sensitivity of OFDM system to the carrier frequency offset (CFO). The main idea is to choose a well-known channel code and to rotate each coordinate by a fixed phase shift such that the maximum inter-carrier interference $(\mathrm{ICI})$ taken over all sub-carriers is minimized. This approach is based on a geometric interpretation of the peak interference to carrier ratio (PICR) of OFDM signals. Simulation results show that a reduction in PICR of $7 \mathrm{~dB}$ can be easily achieved. Furthermore, we addressed the fundamental limit of the proposed technique by providing both an exact and an approximate lower bound of PICR of phase-shifted binary codes. The bounds are applied to some codes: non-redundant binary code, BCH codes, and Reed-Muller codes. Simulation results demonstrated that phase-shift designs approach lower bounds and are resilient to CFO change.
\end{abstract}

Keywords: OFDM, PIRC, Coding

\section{Introduction}

\subsection{Motivations}

Orthogonal frequency-division multiplexing (OFDM) is a multicarrier modulation technique that has been implemented for many high-speed wireless and wireline applications. Indeed, the orthogonal properties among subcarriers of OFDM lead to high spectral efficiency and excellent ability to cope with multipath fading environment. Since the spectra of the subcarriers are overlapping, an accurate frequency-synchronization technique is needed. Due to oscillator inaccuracies and non-ideal transmitter/receiver synchronization, the orthogonal properties are easily broken down and result in errors due to frequency offsets. It is well-known that the carrier frequency offset (CFO) reduces the useful signal power and creates inter-carrier interference (ICI) $[1,2]$.

\subsection{Related works}

Two main approaches have been proposed in the literature to mitigate the ICI problem caused by the CFO. One approach is to estimate and remove the frequency offset [3-6]. In this approach, the frequency-offset estimation is generally performed in two steps: coarse frequencyoffset estimation, which estimates the partial frequency

Correspondence: smida@uic.edu

University of Illinois at Chicago, Chicago, IL, USA offset that is a multiple of the subcarrier spacing and fine frequency-offset estimation, which estimates the remaining part of the offset. There exist several CFO estimation techniques, e.g., training based methods [7-12], and semiblind or blind methods $[3,13]$. Another approach is to use coding ${ }^{1}$ to reduce the sensitivity of the OFDM system to frequency offsets [14-17]. The coding approach is generally adopted after the removal of coarse CFO and hence assumes fine residual CFO. A large range of coding techniques has been developed in recent years and can be classified as follows (see Table 1):

- Self-cancellation: A simple and effective methodknown as the ICI self-cancelation scheme-was proposed by Zhao and Haggman in [18] and later generalized in $[15,19]$, where polynomial coding in the frequency domain is used to mitigate the effect of frequency offset. In this method, copies of the same data symbol are modulated on $r$ adjacent sub-carriers using specific weights. This method can reduce the ICI at the price of lowering the transmission rate by a factor $r$. To further improve the ICI self-cancellation performance, many researchers proposed more efficient mapping (optimized weighting coefficients) for the redundant data symbol modulated over adjacent or non-adjacent subcarriers [20-27]. The weights are designed such that, at the receiver, the ICI at each subcarrier is reduced when a frequency 
Table 1 Coding techniques to reduce OFDM systems sensitivity to frequency shifts

\begin{tabular}{lll}
\hline Coding technique & Complexity & $\begin{array}{l}\text { Transmission-rate } \\
\text { reduction }\end{array}$ \\
\hline Self-cancellation [15, 18-27] & Slight increase & $\geq 2$ \\
Windowing $[30,31]$ & Moderate increase & $\geq 2$ \\
Two-path transmission [32-36] & Slight increase & 2 \\
Correlative coding [37, 38] & Moderate increase & None \\
This paper & Slight Increase & None \\
\hline
\end{tabular}

offset is present. Note that ICI self-cancellation techniques can be regarded as a coding scheme, where only the codewords with low ICI are used, this key idea was first proposed in [28, 29].

- Windowing: Seyedi and Saulnier [30] showed that the polynomial coding in the frequency domain-used by ICI self-cancellation schemes-is equivalent to using windowing in time domain. They hence used windowing at both the transmitter and the receiver to generalize and improve self-cancellation schemes. Recently, Real and Almenar [31] used windowing at the transmitter only allowing a reduction of the receiver complexity without any performance degradation. The windowing methods reduce ICI sensitivity significantly but they lower the transmission rate and increase the complexity of the transceiver.

- Two-path transmission: The two-path transmission method halves the spectral efficiency similar to ICI self-cancellation techniques in [18]. However, unlike ICI self-cancellation method, two-path transmission method transmits the data copies in two concatenated OFDM blocks. The first path represents the standard OFDM signal and the second one is formed by a conjugate of the first path [32, 33]. This approach was generalized in [34-36] by introducing phase rotation and space-time coding.

- Correlative-coding: Contrary to all previous coding techniques, that reduce the sensitivity to CFO at the price of reducing the transmission rate, the correlative coding method do not reduce the data rate $[37,38]$. This method is based on frequency-domain Partial-response Coding (PRC). PRC were originally proposed in the time domain to reduce the sensitivity of single-carrier systems to time offset.

The PRC does not sacrifice spectral efficiency but needs a maximum-likelihood sequence estimator (MLSE) thus increasing the receiver complexity. An interesting combination of correlative-coding and self-cancellation was proposed in [39].

\subsection{Our contributions}

The intend of this paper is to reduce the sensitivity of OFDM systems to CFO without reducing the transmission rate. Our technique is inspired by the following key observation: all current wireless OFDM systems include forward-error-correction or channel codes to reduce the probability of error. Indeed, the forward-error-correction or channel codes have been studied and used for controlling errors in data transmission for the last half century (since Shannon 1948 landmark paper). Sathananthan and Tellambura related ICI reduction to channel coding and showed the capability of any forward-error correction code to reduce the errors caused by the ICI in [14]. We propose here to extend the role of those codes in OFDM systems. The main idea is to rotate each coordinate of the channel code by a fixed phase-shift such that the maximum inter-carrier interference taken over all sub-carriers is minimized. Clearly, the phase-shifted version of the code has the same error correcting capability, rate, decoding complexity as the original code. But it has lower maximum ICI and this reduction comes with no reduction of the spectral efficiency of the system. We proposed a simple techniquephase-shift-to reduce the sensitivity of OFDM system to CFO in [40]. This paper is an extension of this work-it provides an analytical evaluation of the proposed technique.

The key departure of our research from prior works on CFO effect reduction is that (a) we provide a geometric interpretation of the peak interference to carrier ratio (PICR) of OFDM signals, (b) we propose a simple phaseshift approach to reduce the sensitivity of OFDM to fine frequency offset, (c) we analyze the fundamental limit of the proposed technique and obtain a lower bound of the PICR of phase-shifted binary codes which we evaluate for non-redundant binary code, $\mathrm{BCH}$ codes, and Reed-Muller codes, and (d) we numerically evaluate phase-shift designs and prove their resilience to $\mathrm{CFO}$ change.

\section{Statement of the problem}

\subsection{Notations and preliminaries}

An OFDM signal is the sum of many independent signals modulated onto sub-channels of equal bandwidth. The OFDM transmitter applies the inverse discrete Fourier transform (IDFT) operation and sends the symbol sequence to the RF chain. The transmitted signal at time $t$ may be represented as the real part of the complex envelope

$$
S_{\mathbf{c}}(t)=\sum_{n=0}^{N-1} c_{n} \exp \left(j 2 \pi\left(f_{0}+n f_{s}\right) t\right) \quad \text { for } \quad 0 \leq t \leq T_{s},
$$

where $j=\sqrt{-1}, f_{0}$ is the first subcarrier frequency and $f_{s}$ is the bandwidth of each subcarrier. The OFDM-symbol duration $T_{s}$ is equal to $\frac{1}{f_{s}}$ to ensure the orthogonality among the subcarriers. The vector $\mathbf{c}=\left[c_{0}, c_{1}, \ldots, c_{N-1}\right]$ of 
length $N$ is the modulating data sequence. We refer to $\mathbf{c}$ as codeword of code $\mathcal{C}$ that maps blocks of $k$ input bits into blocks of $N$ symbols. The element of the vector $\mathbf{c}, c_{n}$, are typically taken from a constellation $\mathcal{Q}$. In this paper, we assume an equal energy constellation $\mathcal{Q}$ (i.e., all the points in $\mathcal{Q}$ have the same energy such as MPSK). We assume that $S_{\mathbf{c}}(t)$ is transmitted on an additive white Gaussian noise (AWGN) channel. The receiver receives the signal $\mathfrak{R}\left(S_{\mathbf{c}}(t)\right)$ perturbed by noise and performs the inverse operations: the RF chain at the receiver down-converts, processes the received data. The receiver then applies a discrete Fourier transform (DFT) to generate estimate of c. Finally it extracts the block of input bits by applying a suitable error-correction algorithm. The received signal sample for the $k$ th subcarrier after DFT can be written as

$$
\begin{aligned}
y_{k} & =c_{k} b_{0}+\sum_{l=0, l \neq k}^{N-1} b_{l-k} c_{l}+n_{k} \\
& =c_{k} b_{0}+I_{k}+n_{k} \text { for } k=0,1, \ldots, N-1,
\end{aligned}
$$

where $n_{k}$ is a complex Gaussian noise sample. The first term in the right-hand side of (2) represents the desired signal. The ICI term, attributable to the CFO, on the $k$ th subcarrier is expressed by $I_{k}$. The ICI coefficients $b_{k}$ are given by [29]

$$
\begin{aligned}
b_{k} & =\frac{\sin \pi(k+\varepsilon)}{N \sin \frac{\pi}{N}(k+\varepsilon)} \exp \left[j \pi\left(1-\frac{1}{N}\right)(k+\varepsilon)\right], \\
& =\frac{\sin \pi \varepsilon}{N \sin \frac{\pi}{N}(k+\varepsilon)} \exp \left[j \pi\left(1-\frac{1}{N}\right) \varepsilon-j \pi \frac{k}{N}\right],
\end{aligned}
$$

where $\varepsilon$ is the normalized fine frequency offset defined as a ratio between the fine frequency offset and the subcarrier spacing (i.e. $|\varepsilon| \leq \frac{1}{2}$ ). We note that $I_{k}$ is a function of both $\mathbf{c}$ and $\varepsilon$. In the following, we will list the main properties of the the ICI coefficients $b_{k}$. Those properties give insights into the ICI interference $I_{k}$ and will be used in the rest of the paper.

1. Without frequency error $(\varepsilon=0), b_{k}$ reduces to the unit impulse sequence $b_{k}=\delta[k]$.

2. The coefficient $b_{0}$ depends on $\varepsilon$ but is independent of $k$. In other words, all subcarriers experience the same degree of attenuation and rotation of the wanted component.

3. $b_{k}=b_{N+k}$, so if we consider an $N$ modulo numbering $-k$ maps to $N-k$. This property is easy to prove and is used in [20] to propose a repetition code that reduces the ICI.

4. All the coefficients $b_{k}$ for $k \neq 0$ slowly vary with $k$, $b_{k+1} \simeq b_{k}$, the slow varying nature of $b_{k}$ is the key motivation for self ICI cancelation [19]. This property will be used here to derive a more practical (approximate) lower bound of PICR of phase-shifted binary codes.
5. Under the assumption of small normalized frequency offset $(|\varepsilon| \ll 1)$, the coefficients $b_{k}$ became

$$
b_{k} \simeq \frac{\sin \pi \varepsilon}{N \sin \frac{\pi k}{N}} \exp \left[j \pi\left(1-\frac{1}{N}\right) \varepsilon-j \pi \frac{k}{N}\right] .
$$

6. The average power of the inter-carrier interference is derived as [1]

$$
E\left[\left|I_{k}\right|^{2}\right]=\sum_{l=1}^{N-1}\left|b_{l}\right|^{2}=1-\left|b_{0}\right|^{2}
$$

where $E[$.$] denotes the expected value over the$ distribution of data.

\section{Peak interference to carrier ratio evaluation}

In this section, we provide a geometric interpretation of the maximum ICI taken over all sub-carriers that explicitly incorporates the distance between the codewords c and some specific points. Based on this interpretation, we will propose a simple phase-shifted approach to reduce the sensitivity of OFDM systems to CFO.

\subsection{PICR definition}

Sathanannthan and Tellambura $[28,29]$ defined the peak interference to carrier ratio (PICR) as the maximum interference-to-signal ratio for any subcarrier:

$$
\operatorname{PICR}(\mathbf{c}, \varepsilon)=\max _{0 \leq k \leq N-1} \frac{\left|I_{k}\right|^{2}}{\left|b_{0} c_{k}\right|^{2}} \text {. }
$$

It specifies the worst-case ICI on any subcarrier. Large $I_{k}$ value cause high bit errors in subcarriers. Therefore, to reduce ICI effects, PICR should be minimized [29]. Here we also define the PICR of a code $\mathcal{C}$ as the maximum PICR over all codewords in $\mathcal{C}$

$$
\operatorname{PICR}(\mathcal{C}, \varepsilon)=\max \{\operatorname{PICR}(\mathbf{c}, \varepsilon) \quad \mid \quad \forall \mathbf{c} \in \mathcal{C}\} .
$$

Note that the expression of PICR is similar to the peakto-mean envelope power ratio (PMEPR) that is considered as measure of the fluctuation of the OFDM signal ${ }^{2}$ $\operatorname{PMEPR}(\mathbf{c})=\max _{0 \leq t \leq 1} \frac{\left|S_{\mathbf{c}}(t)\right|^{2}}{\|\mathbf{c}\|^{2}}$.

\subsection{PICR geometric representation}

We rewrite $\operatorname{PICR}(\mathcal{C}, \varepsilon)$ as

$$
\operatorname{PICR}(\mathcal{C}, \varepsilon)=\max _{0 \leq k \leq N-1} \mathcal{J}(k, \varepsilon), \text { where } \mathcal{J}(k, \varepsilon)=\max _{\mathbf{c} \in \mathcal{C}} \frac{\left|I_{k}\right|^{2}}{\left|b_{0} c_{k}\right|^{2}} .
$$

We refer to $\mathcal{J}(k, \varepsilon)$ as the maximum interference-tosignal ratio of code $\mathcal{C}$ on subcarrier $k$ and focus on its computation. The next theorem provides a geometric interpretation of the PICR per subcarrier [40].

Theorem 3.1 Let $\mathcal{C}$ be a code of length $N$ defined over unit energy constellation $Q$. For each $k$, the value of $\mathcal{J}(k, \varepsilon)$ 
is determined by the distance between the codewords $\mathbf{c} \in \mathcal{C}$ and the points $\mathbf{w}_{\mathbf{k}}$ and $j \mathbf{w}_{\mathbf{k}}$, where

$$
\mathbf{w}_{\mathbf{k}}=\left[b_{-k}^{*}, \ldots, b_{-1}^{*}, 0, b_{1}^{*}, \ldots, b_{N-1-k}^{*}\right] .
$$

If $\mathcal{C}$ is a cyclic code then

$$
\operatorname{PICR}(\mathcal{C}, \varepsilon)=\mathcal{J}(0, \varepsilon) \text {. }
$$

The value of $\mathcal{J}(0, \varepsilon)$ is determined by the distance between the codewords $\mathbf{c} \in \mathcal{C}$ and the two points $\mathbf{w}_{\mathbf{0}}$ and $j \mathbf{w}_{\mathbf{0}}$.

Proof For any vectors $\mathbf{x}=\left(x_{0}, x_{1}, \ldots, x_{N-1}\right)$ and $\mathbf{y}=$ $\left(y_{0}, y_{1}, \ldots, y_{N-1}\right)$, let $\mathbf{x . y}=\sum_{i=0}^{N-1} x_{i} y_{i}^{*}$ denote the inner product of $\mathbf{x}$ and $\mathbf{y}$. Using the identities

$$
\begin{aligned}
\|\mathbf{x}-\mathbf{y}\|^{2} & =\|\mathbf{x}\|^{2}+\|\mathbf{y}\|^{2}-2 \mathfrak{R}(\mathbf{x} . \mathbf{y}), \\
\|\mathbf{x}-j \mathbf{y}\|^{2} & =\|\mathbf{x}\|^{2}+\|\mathbf{y}\|^{2}-2 \mathfrak{I}(\mathbf{x} . \mathbf{y}),
\end{aligned}
$$

we derive the following equalities

$$
\begin{aligned}
\left\|\mathbf{c}-\mathbf{w}_{\mathbf{k}}\right\|^{2} & =\|\mathbf{c}\|^{2}+\left\|\mathbf{w}_{\mathbf{k}}\right\|^{2}-2 \Re\left(I_{k}\right), \\
\left\|\mathbf{c}-j \mathbf{w}_{\mathbf{k}}\right\|^{2} & =\|\mathbf{c}\|^{2}+\left\|\mathbf{w}_{\mathbf{k}}\right\|^{2}-2 \Im\left(I_{k}\right) .
\end{aligned}
$$

where $\mathbf{c}=\left[c_{0}, \ldots, c_{N-1}\right]$. Without loss of generality, we assume that the elements of $Q$ have unit amplitude $\left(\left|c_{n}\right|^{2}=1,0 \leq n \leq N-1\right)$ then $\|\mathbf{c}\|^{2}=N .^{3}$

Using properties 3 and 6 of the ICI coefficients, we get $\left\|\mathbf{w}_{\mathbf{k}}\right\|^{2}=\sum_{l=-k, l \neq 0}^{N-1-k}\left|b_{l}\right|^{2}=\sum_{l=1}^{N-1}\left|b_{l}\right|^{2}=1-\left|b_{0}\right|^{2}$. Hence, we rewrite $\mathcal{J}(k, \varepsilon)$ as follows:

$$
\begin{aligned}
\mathcal{J}(k, \varepsilon) & =\max _{\mathbf{c} \in \mathcal{C}} \frac{\left|I_{k}\right|^{2}}{\left|b_{0}\right|^{2}}=\max _{\mathbf{c} \in \mathcal{C}} \frac{1}{\left|b_{0}\right|^{2}}\left[\left(\Re\left(I_{k}\right)\right)^{2}+\left(\mathfrak{I}\left(I_{k}\right)\right)^{2}\right], \\
& =\max _{\mathbf{c} \in \mathcal{C}} \frac{\left(N+1-\left|b_{0}\right|^{2}-\left\|\mathbf{c}-\mathbf{w}_{\mathbf{k}}\right\|^{2}\right)^{2}+\left(N+1-\left|b_{0}\right|^{2}-|| \mathbf{c}-j \mathbf{w}_{\mathbf{k}} \|^{2}\right)^{2}}{4\left|b_{0}\right|^{2}} .
\end{aligned}
$$

Let $\mathbf{c}^{+}$denote the codeword for which $\mathcal{J}(k, \varepsilon)$ is attained, $\mathbf{c}^{+}$maximizes

$$
\begin{aligned}
& \left(N+1-\left|b_{0}\right|^{2}-\left\|\mathbf{c}-\mathbf{w}_{\mathbf{k}}\right\|^{2}\right)^{2} \\
& +\left(N+1-\left|b_{0}\right|^{2}-\left\|\mathbf{c}-j \mathbf{w}_{\mathbf{k}}\right\|^{2}\right)^{2},
\end{aligned}
$$

among all the codewords. So for each $k$, the value of $\mathcal{J}(k, \varepsilon)$ is determined by the distance between the codewords $\mathbf{c} \in \mathcal{C}$ and the points $\mathbf{w}_{\mathbf{k}}$ and $j \mathbf{w}_{\mathbf{k}}$.

The proof of the second part of Theorem 3.1 is based on the definition of cyclic code. For every codeword $\mathbf{c}=$ $\left[c_{0}, \ldots, c_{N-1}\right] \in$ cyclic code $\mathcal{C}$, the word $\left[c_{-k}, \ldots, c_{N-k-1}\right]$ obtained by a cyclic shift of $k$ components is again a codeword. In addition, based on property 3 of the coefficients $b_{n}, \mathbf{w}_{\mathbf{k}}$ is obtained by $k$ left cyclic shift of the element of $\mathbf{w}_{\mathbf{0}}$. If we assume that the $\operatorname{PICR}(\mathcal{C}, \varepsilon)$ is reached for the subcarrier $k \neq 0$ and code $\mathbf{c}^{k+}$, then we have just to shift the codeword $\mathbf{c}^{k+}$ by $k$ to find a codeword $\mathbf{c}^{0+}$ and it is easy to prove that the interference generated for the subcarrier 0 and code $\mathbf{c}^{0+}$ is equals to $\operatorname{PICR}(\mathcal{C}, \varepsilon)$. Hence, the $\operatorname{PICR}(\mathcal{C}, \varepsilon)$ is also reached for the subcarrier $k=0$.
The geometric interpretation of Theorem 3.1 is simple but important because it explicitly states that the value of $\operatorname{PICR}(\mathcal{C}, \varepsilon)$ is determined by the distance between the codewords $\mathbf{c} \in \mathcal{C}$ and the points $\mathbf{w}_{\mathbf{k}}$ and $j \mathbf{w}_{\mathbf{k}}, k=$ $0, \ldots, N-1$. Furthermore for cyclic code, the value of PICR is only function of the distance between the codewords and the two points $\mathbf{w}_{\mathbf{0}}$ and $j \mathbf{w}_{\mathbf{0}}$. This representation leads to an understanding of the value of $\operatorname{PICR}(\mathcal{C}, \varepsilon)$ and how to reduce it through phase-shift approach which is described in the following Section 4.

\section{PICR reduction}

\subsection{Phase-shift technique: illustration}

Theorem 3.1 provides a geometric interpretation of the value of PICR. Based on this interpretation, we propose a simple approach that was originally proposed by Jones and Wilkinson [41, 42] and further developed by Tarokh, Jafarkhani and Paterson $[43,44]$ to reduce PMEPR. The idea is to take a code with good BER performance and to rotate each coordinate of the code by a fixed phase-shift such that the maximum PICR evaluated for all codewords is minimized. For the purpose of illustration, we assume a unit amplitude constellation; thus all the codewords are points on the $N$-dimensional complex sphere of radius $\sqrt{N}$. The phase-shifts may reduce the PICR by reducing/increasing the distance between the codewords and the points $\mathbf{w}_{\mathbf{k}}$ and $j \mathbf{w}_{\mathbf{k}}, k=0, \ldots, N-1$. For cyclic code, we just focus on the distance between the codewords and the points $\mathbf{w}_{\mathbf{0}}$ and $j \mathbf{w}_{\mathbf{0}}$ only. Our design will shift the codewords without changing the distance between them as illustrated in Fig. 1. So it leaves the error-correcting properties of the code unchanged. In addition, we can use the standard decoding algorithm of the original code after the received signals are rotated back.

The reduction of interference for OFDM can now be tackled as follows: For a given $\operatorname{code} \mathcal{C}$, the reducing phaseshift problem is to find offset $\mathbf{v}=\left[v_{0}, \ldots, v_{N-1}\right] \in \mathbb{Z}_{2^{h}}^{N}$ such that $\operatorname{PICR}\left(\mathcal{C}_{v}, \epsilon\right)$ is minimized, where $\mathcal{C}_{v}=\left\{e^{j \pi \mathbf{v} / 2^{h}} \mathbf{c} \mid \mathbf{c} \in\right.$ $\mathcal{C}\}, h$ is an integer that set the resolution of the offset. Notice that, when $h$ tends to infinity, we can consider $\operatorname{PICR}\left(\mathcal{C}_{v}, \epsilon\right)$ as a continuous multi-variable function of $\phi_{0}=2 \pi v_{0} / 2^{h}, \ldots, \phi_{N-1}=2 \pi v_{N-1} / 2^{h}$. Because of the continuity, the global minimum of this function over $[0,2 \pi] \times[0,2 \pi] \times \cdots \times[0,2 \pi]$ exists. But this function may have various local minima and saddle points over this compact set as well. There are various methods for minimization of $\operatorname{PICR}\left(\mathcal{C}_{v}, \epsilon\right)$ in the literature, but they can only guarantee convergence to a local minima. As representative of these methods, we have used the gradient method in our simulations. It is important to note that this approach can be applied to binary and non-binary codes. Then, we used the lower bound, derived in the following section, to evaluate the optimality of our design. 


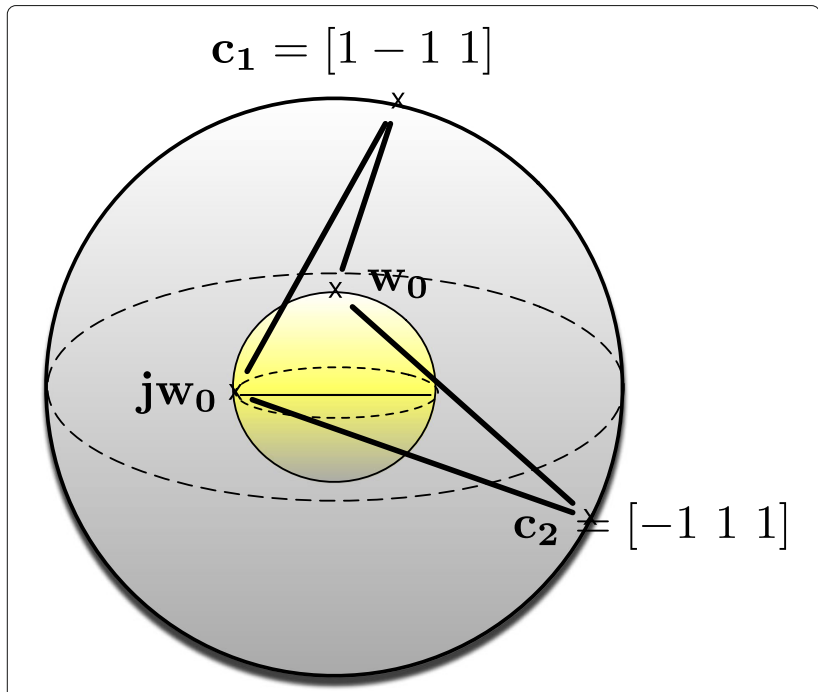

Fig. 1 Illustration of the geometric interpretation of PICR. We plot two codewords on the sphere $N=3$. By rotating the codewords over the sphere (through phase-shift) we can change the distance between the codewords and the two points $\mathbf{w}_{\mathbf{0}}$ and $\mathbf{j} \mathbf{w}_{\mathbf{0}}$

\subsection{Phase-shift technique: range}

This section is dedicated to prove that phase-shift designs are robust to CFO change. The optimum offset $\mathbf{v}$ minimizes the PICR for a range of frequency offset (not for a specific frequency offset value). To do so we need the following proposition.

Proposition 4.1 Let $\mathcal{C}$ be a code of length $N$ defined over an equal energy constellation $Q$. Let $\varepsilon_{1}$ and $\varepsilon_{2}$ be small normalized frequency offsets $\left(\left|\varepsilon_{1}\right| \ll 1\right.$ and $\left.\left|\varepsilon_{2}\right| \ll 1\right)$. If the offset $\mathbf{v} \in \mathbb{Z}_{2^{h}}^{N}$ minimizes $\operatorname{PICR}\left(\mathcal{C}_{v}, \varepsilon_{1}\right)$ that it minimizes $\operatorname{PICR}\left(\mathcal{C}_{v}, \epsilon_{2}\right)$ too.

Proof The proof is based on the fifth property of the coefficients $b_{n}$. We rewrite the $\operatorname{PICR}\left(\mathcal{C}, \varepsilon_{2}\right)$ as

$$
\operatorname{PICR}\left(\mathcal{C}, \varepsilon_{2}\right)=\max _{0 \leq k \leq N-1, \mathbf{c} \in \mathcal{C}} \frac{\left|I_{k}\left(\varepsilon_{2}\right)\right|^{2}}{\left|b_{0}\left(\varepsilon_{2}\right) c_{k}\right|^{2}}
$$

where $I_{k}\left(\varepsilon_{2}\right)$ is the inter-carrier interference on the $k$ th subcarrier and $b_{0}\left(\varepsilon_{2}\right)$ is the ICI coefficient on the desired signal evaluated for normalized CFO of $\varepsilon_{2}$ as in Eq. (2). Using the property 5 of the ICI coefficients, we have

$$
\begin{aligned}
\operatorname{PICR}\left(\mathcal{C}, \varepsilon_{2}\right) & =\max _{0 \leq k \leq N-1, \mathbf{c} \in \mathcal{C}} \frac{\left|I_{k}\left(\varepsilon_{2}\right)\right|^{2}}{\left|b_{0}\left(\varepsilon_{2}\right) c_{k}\right|^{2}}, \\
& \simeq \frac{\left|\sin \left(\pi \varepsilon_{2}\right) b_{0}\left(\varepsilon_{2}\right)\right|^{2}}{\left|\sin \left(\pi \varepsilon_{1}\right) b_{0}\left(\varepsilon_{1}\right)\right|^{2}} \max _{0 \leq k \leq N-1, \mathbf{c} \in \mathcal{C}} \frac{\left|I_{k}\left(\varepsilon_{1}\right)\right|^{2}}{\left|b_{0}\left(\varepsilon_{1}\right) c_{k}\right|^{2}}, \\
& =\left|\frac{\sin \left(\pi \varepsilon_{2}\right) b_{0}\left(\varepsilon_{2}\right)}{\sin \left(\pi \varepsilon_{1}\right) b_{0}\left(\varepsilon_{1}\right)}\right|^{2} \operatorname{PICR}\left(\mathcal{C}, \varepsilon_{1}\right) .
\end{aligned}
$$

Hence, the offset $\mathbf{v} \in \mathbb{Z}_{2^{h}}^{N}$, that minimizes $\operatorname{PICR}\left(\mathcal{C}_{v}, \varepsilon_{1}\right)$, minimizes $\operatorname{PICR}\left(\mathcal{C}_{v}, \epsilon_{2}\right)$ too. Therefore, there is no need to search for an offset $\mathbf{v}$ specific to each carrier frequency offset. The simulation results will show in Section 6 that the optimum set of shifts, derived for $\varepsilon=0.1$, reduce the inter-carrier interference for a wide range of CFO $(0<\varepsilon<0.5)$.

\section{Minimum PICR for phase-shifted binary code}

Motivated by the previous design of phase-shifts, we would like to know how much PICR reduction can be achieved. In particular, in our framework, similar to that of Schmidt's [45], we consider the following question. For a given binary code $\mathcal{B}$, let $\mathcal{B}_{v}$ be the phase-shifted version of $\mathcal{B}$, what is

$$
\min _{\mathcal{B}_{v} \in E_{h}(B)} \operatorname{PICR}\left(\mathcal{B}_{v}, \epsilon\right)
$$

We provide a lower bound and an approximated lower bound for this limit. The bounds are used to evaluate the optimality of our design.

\subsection{Main results}

Our lower bound in the spirit of Schmidt's [45] will be expressed in terms of the covering radius of the binary code $\mathcal{B}$ defined below.

Definition The covering radius of a code $\mathcal{B} \subseteq\{1,-1\}^{N}$ is defined to be

$$
\rho(\mathcal{B}) \triangleq \max _{x \in \mathbb{Z}_{2}^{N}} \min _{\mathbf{b}^{+} \in \mathcal{B}^{+}} w t_{H}(b+x) .
$$

where each codeword $\mathbf{b}^{+} \in \mathcal{B}^{+}$is associated with a codeword $\mathbf{b} \in \mathcal{B}$ (where $\left.b_{i}^{+}=\frac{b_{i}+1}{2}\right), w t_{H}(b)$ is the Hamming weight of a binary vector $b$. We now state our main result.

Theorem 5.1 Given a binary code $B \subseteq\{1,-1\}^{N}$ with covering radius $\rho(\mathcal{B})$, then if $h>1$

$$
\begin{aligned}
& \min _{C \in E_{h}(B)} \operatorname{PICR}(\mathcal{C}, \epsilon) \geq \\
& \frac{1}{8\left|b_{0}\right|^{2}}\left[2 N-2\left|b_{0}\right|^{2}-2 \sum_{i=1}^{N-1}\left(1-\left|b_{i}\right|\right)^{2}-\lambda(N+2 \rho(\mathcal{B}))\right]^{+^{2}},
\end{aligned}
$$

where $\lambda=b_{\max } 2^{2 h-2} \sin ^{2}\left(\frac{\pi}{2^{h}}\right), b_{\max }=\max _{i \neq 0}\left|b_{i}\right|$ and $[x]^{+}=\max (x, 0)$.

If $|\epsilon| \ll 1$ then

$$
\begin{aligned}
& \min _{C \in E_{h}(B)} \operatorname{PICR}(\mathcal{C}, \epsilon) \gtrsim \\
& \frac{1}{8\left|b_{0}\right|^{2}}\left[2 N-2\left|b_{0}\right|^{2}-2 \sum_{i=1}^{N-1}\left(1-\left|b_{i}\right|\right)^{2}-\lambda_{\text {avg }}(N+2 \rho(\mathcal{B}))\right]^{+}
\end{aligned}
$$


where $\lambda_{\mathrm{avg}}=b_{\mathrm{avg}} 2^{2 h-2} \sin ^{2}\left(\frac{\pi}{2^{h}}\right)$ and $b_{\mathrm{avg}}=$ $\operatorname{mean}_{1 \leq i \leq N-1}\left|b_{i}\right|$.

The proof of Theorem 5.1 is provided in Appendix. The preceding lower bound is a decreased function of $h$. The corollary below states the asymptotic lower bound for $h \rightarrow \infty$.

Corollary 5.2 Given a binary code $B \subseteq\{1,-1\}^{N}$ with covering radius $\rho(\mathcal{B})$, then if $h>1$

$$
\begin{aligned}
& \min _{C \in E_{\infty}(B)} \operatorname{PICR}(\mathcal{C}, \epsilon) \geq \\
& \frac{1}{8\left|b_{0}\right|^{2}}\left[2 N-2\left|b_{0}\right|^{2}-2 \sum_{i=1}^{N-1}\left(1-\left|b_{i}\right|\right)^{2}-b_{\max } \frac{\pi^{2}}{4}(N+2 \rho(\mathcal{B}))\right]^{+^{2}} .
\end{aligned}
$$

If $|\epsilon| \ll 1$ then

$\min _{C \in E_{\infty}(B)} \operatorname{PICR}(\mathcal{C}, \epsilon) \gtrsim$

$\frac{1}{8\left|b_{0}\right|^{2}}\left[2 N-2\left|b_{0}\right|^{2}-2 \sum_{i=1}^{N-1}\left(1-\left|b_{i}\right|\right)^{2}-b_{\text {avg }} \frac{\pi^{2}}{4}(N+2 \rho(\mathcal{B}))\right]^{+^{2}}$.

Proof Similarly to [45], we used $\lim _{h \rightarrow \infty} 2^{2 h-2} \sin ^{2}\left(\frac{\pi}{2^{h}}\right)=\frac{\pi^{2}}{4}$.

\subsection{Examples}

\subsubsection{Non-redundant BPSK signaling}

The code $\{1,-1\}^{N}$ is a binary code without redundancy, $\rho\left(\{1,-1\}^{N}\right)=0$, Theorem 5.1 and Corollary 5.2 imply the following.

Corollary 5.3 For $h>1$, the PICR of a $2^{h}$-ary PSK code equivalent to $\{1,-1\}^{N}$ is at least

$$
\begin{aligned}
& \min _{C \in E_{h}\left(\mathbb{Z}_{2}^{N}\right)} \operatorname{PICR}(\mathcal{C}, \epsilon) \gtrsim \\
& \frac{1}{8\left|b_{0}\right|^{2}}\left[2 N-2\left|b_{0}\right|^{2}-2 \sum_{i=1}^{N-1}\left(1-\left|b_{i}\right|\right)^{2}-\lambda_{\text {avg }} N\right]^{+^{2}}
\end{aligned}
$$

and

$$
\begin{aligned}
& \min _{C \in E_{\infty}\left(\mathbb{Z}_{2}^{N}\right)} \operatorname{PICR}(\mathcal{C}, \epsilon) \gtrsim \\
& \frac{1}{8\left|b_{0}\right|^{2}}\left[2 N-2\left|b_{0}\right|^{2}-2 \sum_{i=1}^{N-1}\left(1-\left|b_{i}\right|\right)^{2}-b_{\text {avg }} \frac{\pi^{2}}{4} N\right]^{+^{2}} .
\end{aligned}
$$

For $N=16$ and $N=64$ the PICR approximated lower bounds are 0.10 and 0.22 , respectively. Since the PICR for non-redundant binary code are $0.19(N=16)$ and 0.50 $(N=64)$, the maximum PICR reduction of the proposed phase-shift designs is $3 \mathrm{~dB}$ for non-redundant code.

\subsubsection{Reed-Muller codes}

In the following we inspect codes that are equivalent to first order Reed-Muller codes of length $2^{m}, R M(1, m)$.

Corollary 5.4 For $h>1$ and $N=2^{m}$, the PICR of $a$ $2^{h}$-ary PSK code equivalent to $R M(1, m)$ is at least

$$
\begin{array}{r}
\min _{C \in E_{h}(R M(1, m))} \operatorname{PICR}(\mathcal{C}, \epsilon) \gtrsim \frac{1}{8\left|b_{0}\right|^{2}}\left[2 N-2\left|b_{0}\right|^{2}-2 \sum_{i=1}^{N-1}\left(1-\left|b_{i}\right|\right)^{2}\right. \\
\left.\left.-\lambda_{\text {avg }}\left(N+\left(2^{m}-2^{m / 2}\right)\right)\right)\right]^{+^{2}},
\end{array}
$$

and when $h=\infty$

$$
\begin{aligned}
\min _{C \in E_{\infty}(R M(1, m))} \operatorname{PICR}(\mathcal{C}, \epsilon) \gtrsim \frac{1}{8\left|b_{0}\right|^{2}}[ & 2 N-2\left|b_{0}\right|^{2}-2 \sum_{i=1}^{N-1}\left(1-\left|b_{i}\right|\right)^{2} \\
& \left.\left.-b_{\text {avg }} \frac{\pi^{2}}{4}\left(N+\left(2^{m}-2^{m / 2}\right)\right)\right)\right] .
\end{aligned}
$$

Proof It was proved in [46] that

$$
2^{m-1}-2^{(m-1) / 2} \leq \rho(R M(1, m)) \leq 2^{m-1}-2^{m / 2-1} .
$$

\subsubsection{Binary primitive $\mathrm{BCH}$ codes}

Next we present the lower bounds on the PICR of codes that equivalent to binary primitive $t$-error-correcting $\mathrm{BCH}$ codes, $\mathcal{B}(t, m)$.

Corollary 5.5 For $h>1$ and $N=2^{m}-1$, the PICR of a $2^{h}$-ary PSK code equivalent to t-error-correcting $B C H$ codes, $\mathcal{B}(t, m)$ is at least

$$
\begin{aligned}
& \min _{C \in E_{h}(\mathcal{B}(t, m))} \operatorname{PICR}(\mathcal{C}, \epsilon) \gtrsim \\
& \left.\frac{1}{8\left|b_{0}\right|^{2}}\left[2 N-2\left|b_{0}\right|^{2}-2 \sum_{i=1}^{N-1}\left(1-\left|b_{i}\right|\right)^{2}-\lambda_{\text {avg }}(N+(4 t-2))\right)\right]^{+},
\end{aligned}
$$

and when $h=\infty$

$$
\begin{aligned}
& \underset{C \in E_{\infty}(\mathcal{B}(t, m))}{\operatorname{PICR}(\mathcal{C}, \epsilon) \gtrsim} \\
& \left.\frac{1}{8\left|b_{0}\right|^{2}}\left[2 N-2\left|b_{0}\right|^{2}-2 \sum_{i=1}^{N-1}\left(1-\left|b_{i}\right|\right)^{2}-b_{\text {avg }} \frac{\pi^{2}}{4}(N+(4 t-2))\right)\right]^{+2} .
\end{aligned}
$$

Proof The authors of [47] proved that there exists an $m_{0}$ depending on $t$ such that $\rho(\mathcal{B}(t, m))=2 t-1$ for all $m \geq$ $m_{o}$.

\section{Numerical results}

The numerical results presented in this section aim to evaluate the ICI reduction performance of the proposed phase-shift approach. We considered a normalized frequency offset of $\varepsilon=0.1$ and two well-known codes extended Bose-Chaudhuri-Hocqenghem $(\mathrm{BCH})$ and Reed 
Table 2 Optimum phase shifts for $\mathrm{BCH}(64,10)$ in radians

\begin{tabular}{llllllll}
\hline$\phi_{0}=2.4683$ & $\phi_{1}=1.7502$ & $\phi_{2}=4.4962$ & $\phi_{3}=4.0882$ & $\phi_{4}=0.1253$ & $\phi_{5}=2.9330$ & $\phi_{6}=5.3401$ & $\phi_{7}=2.2695$ \\
$\phi_{8}=4.2003$ & $\phi_{9}=4.0068$ & $\phi_{10}=6.2288$ & $\phi_{11}=5.6747$ & $\phi_{12}=5.3153$ & $\phi_{13}=1.0958$ & $\phi_{14}=4.5622$ & $\phi_{15}=6.0023$ \\
$\phi_{16}=3.2120$ & $\phi_{17}=1.5937$ & $\phi_{18}=4.9119$ & $\phi_{19}=3.7611$ & $\phi_{20}=4.1274$ & $\phi_{21}=5.8449$ & $\phi_{22}=5.5075$ & $\phi_{23}=5.4723$ \\
$\phi_{24}=4.0787$ & $\phi_{25}=4.3756$ & $\phi_{26}=4.4991$ & $\phi_{27}=3.1733$ & $\phi_{28}=3.6295$ & $\phi_{29}=2.6383$ & $\phi_{30}=5.2078$ & $\phi_{31}=5.7397$ \\
$\phi_{32}=3.4149$ & $\phi_{33}=0.9170$ & $\phi_{34}=0.8856$ & $\phi_{35}=5.4017$ & $\phi_{36}=5.2185$ & $\phi_{37}=2.2959$ & $\phi_{38}=0.6368$ & $\phi_{39}=1.1671$ \\
$\phi_{40}=3.1718$ & $\phi_{41}=2.9789$ & $\phi_{42}=5.0463$ & $\phi_{43}=5.1208$ & $\phi_{44}=0.9384$ & $\phi_{45}=3.8918$ & $\phi_{46}=2.3587$ & $\phi_{47}=2.3106$ \\
$\phi_{48}=2.7175$ & $\phi_{49}=3.2082$ & $\phi_{50}=0.9428$ & $\phi_{51}=1.0826$ & $\phi_{52}=5.4491$ & $\phi_{53}=5.7840$ & $\phi_{54}=4.6229$ & $\phi_{55}=1.2202$ \\
$\phi_{56}=3.5798$ & $\phi_{57}=0.1929$ & $\phi_{58}=2.7888$ & $\phi_{59}=5.5207$ & $\phi_{60}=1.8680$ & $\phi_{61}=4.5057$ & $\phi_{62}=0.4838$ & $\phi_{63}=3.2663$ \\
\hline
\end{tabular}

Muller (RM). We note that Pyndiah [48] has proposed a simple soft-input soft-output (approximated MAP) decoder for $\mathrm{BCH}$ codes, based on Chase algorithm, that gives excellent BER performance. RM codes achieve capacity on erasure channels and reduce PMEPR $[49,50]$.

For the BPSK case, we have optimized the phase-shifts by finding a local minima of $\operatorname{PICR}(\mathcal{C}, \varepsilon)$. Once the optimal phase-shifts were computed, they were rounded to 8-PSK, and 16-PSK phase-shifts. The PICR was then calculated for the rounded phase shifted. The optimal set of shifts for $\mathrm{BCH}(64,10)$ are given in Table 2. The reduction in PICR is $2.56 \mathrm{~dB}$, it decreases to 1.99 and $1.39 \mathrm{~dB}$ for 16 PSK and 8-PSK, respectively. The optimal set of shifts for $\mathrm{RM}(1,6)$ are given in Table 3. The reduction in PICR is $7.10 \mathrm{~dB}$; it decreases to 6.28 and $5.86 \mathrm{~dB}$ for 16-PSK and 8PSK, respectively. The numerical results show that a fixed phase-shift applied to code coordinates reduces the ICI, and that most of the gain can be obtained by using 8PSK and 16-PSK type phase-shifts. The phase-shift design reduce the PICR from $0.255(\mathrm{BCH})$ and $0.519(\mathrm{RM})$ to 0.14 and 0.098 , respectively. These values approaches the lower bound in Corollary 5.4 and Corollary 5.5.

Furthermore, we use the same set of phase-shifts with various CFO $(0<\varepsilon<0.5)$. The PICR results shown in Figs. 2 and 3 prove that the optimum set of shifts, derived for $\varepsilon=0.1$, reduce the inter-carrier interference for a wide range of CFO. For the purpose of comparison, we also plot the PICR with a self-interference cancellation that reduces the transmission-rate by half [18]. In fairness, we implemented the self-cancellation technique over the non-redundant data sequences (no channel coding). The simulation results prove that our simple phase-shift designs are very competitive - especially when applied to codes with high large covering radius like RM codes. To illustrate the impact of our phase-shift technique on the bit error rate (BER), we consider $\operatorname{RM}(1,6)$ code and BPSK modulation. The BER vs. signal to noise ratio (SNR) performance with and without phase-shifts are shown in Fig. 4. Note that the phase-shift technique improves the performance at BER $10^{-6}$ by $10 \mathrm{~dB}$ for normalized frequency offset $\epsilon=0.1$.

\section{Conclusions}

In this paper, we proposed an interference reduction scheme to mitigate the effects of CFO in OFDM system. We provided a geometrical interpretation of $\operatorname{PICR}(\mathcal{C}, \varepsilon)$, the PICR of $\mathcal{C}$. To reduce PICR, we focused on a simple phase-shift approach. The idea is to take a code with good BER performance and to rotate each coordinate of the code by a fixed phase-shift such that the maximum PICR taken over all codewords is minimized. This shift leaves unchanged the error-correcting properties of the code. In addition, we can use the standard decoding algorithm of the original code after back rotation of the received signals. Simulation results show that a reduction of $7 \mathrm{~dB}$ can freely be obtained.

We also study the fundamental limit of the proposed technique by providing an approximated lower bound of the PICR. The bound is applied to some codes: nonredundant binary code, $\mathrm{BCH}$ codes, and Reed-Muller

Table 3 Optimum phase shifts for $\operatorname{RM}(1,6)$ in radians

\begin{tabular}{llllllll}
\hline$\phi_{0}=1.5620$ & $\phi_{1}=4.5390$ & $\phi_{2}=3.5491$ & $\phi_{3}=0.3789$ & $\phi_{4}=2.9522$ & $\phi_{5}=4.9135$ & $\phi_{6}=1.9776$ & $\phi_{7}=3.7244$ \\
$\phi_{8}=3.7291$ & $\phi_{9}=5.2304$ & $\phi_{10}=3.8296$ & $\phi_{11}=2.8186$ & $\phi_{12}=5.5612$ & $\phi_{13}=4.4629$ & $\phi_{14}=3.4743$ & $\phi_{15}=6.1346$ \\
$\phi_{16}=4.7435$ & $\phi_{17}=1.3264$ & $\phi_{18}=3.1416$ & $\phi_{19}=0.1649$ & $\phi_{20}=4.9018$ & $\phi_{21}=2.6437$ & $\phi_{22}=5.8063$ & $\phi_{23}=0.9283$ \\
$\phi_{24}=4.0979$ & $\phi_{25}=3.9276$ & $\phi_{26}=5.6677$ & $\phi_{27}=5.7450$ & $\phi_{28}=3.8378$ & $\phi_{29}=1.3179$ & $\phi_{30}=4.9345$ & $\phi_{31}=5.4689$ \\
$\phi_{32}=0.0817$ & $\phi_{33}=3.2805$ & $\phi_{34}=2.8142$ & $\phi_{35}=5.0262$ & $\phi_{36}=5.0639$ & $\phi_{37}=1.0568$ & $\phi_{38}=0.8457$ & $\phi_{39}=2.9594$ \\
$\phi_{40}=5.7340$ & $\phi_{41}=5.5270$ & $\phi_{42}=3.2478$ & $\phi_{43}=3.5318$ & $\phi_{44}=1.1401$ & $\phi_{45}=1.7986$ & $\phi_{46}=2.3697$ & $\phi_{47}=0.3248$ \\
$\phi_{48}=4.5588$ & $\phi_{49}=1.6792$ & $\phi_{50}=0.0914$ & $\phi_{51}=6.2392$ & $\phi_{52}=1.8721$ & $\phi_{53}=1.1146$ & $\phi_{54}=3.4850$ & $\phi_{55}=6.2436$ \\
$\phi_{56}=1.3990$ & $\phi_{57}=5.3068$ & $\phi_{58}=0.0079$ & $\phi_{59}=6.0174$ & $\phi_{60}=4.3187$ & $\phi_{61}=4.3175$ & $\phi_{62}=6.1484$ & $\phi_{63}=5.8044$ \\
\hline
\end{tabular}




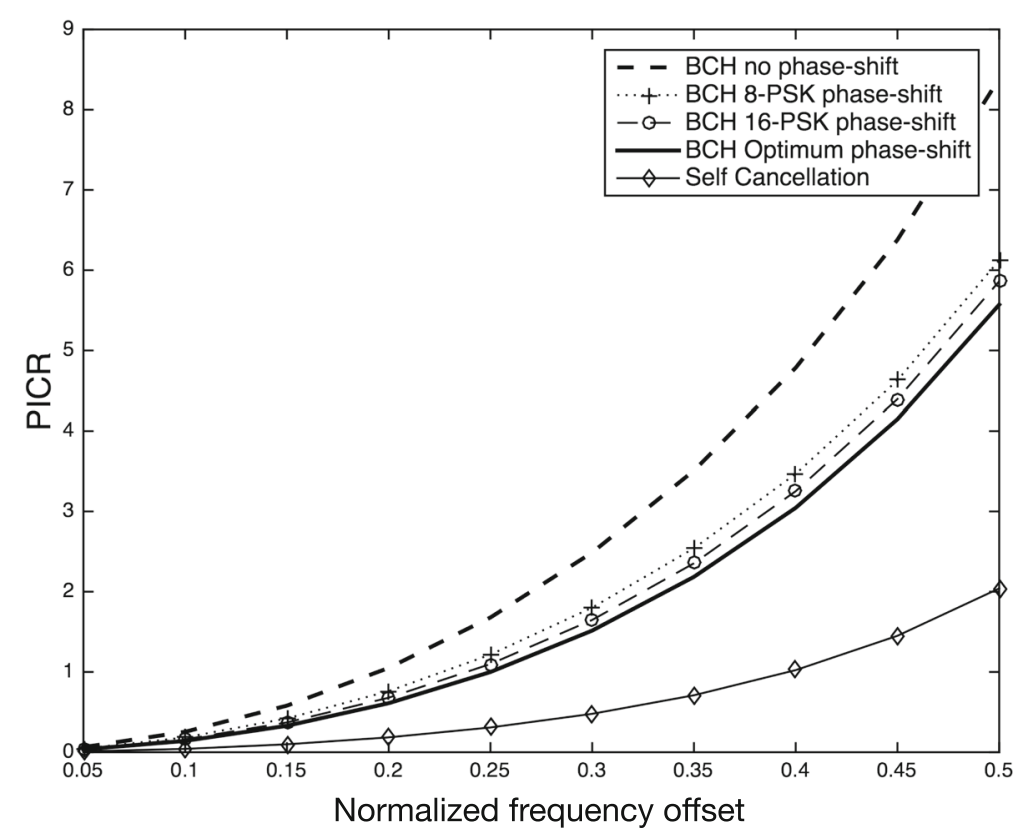

Fig. 2 PICR of $2^{h}$-ary PSK code equivalent to $\mathrm{BCH}(64,10)$

codes. It is demonstrated that our phase-shift designs approach our bound.

\section{Endnotes}

${ }^{1}$ One more approach uses signal processing, such as time and frequency equalization, to reduce the sensitivity of OFDM systems to frequency offset. Although efficient, signal processing techniques usually increase the complexity of the transceiver (see [16] and references therein).

${ }^{2}$ The authors of [29] have noted that despite the similarities between PICR and PMEPR, the PICR problem differs from PMEPR issue in several ways.

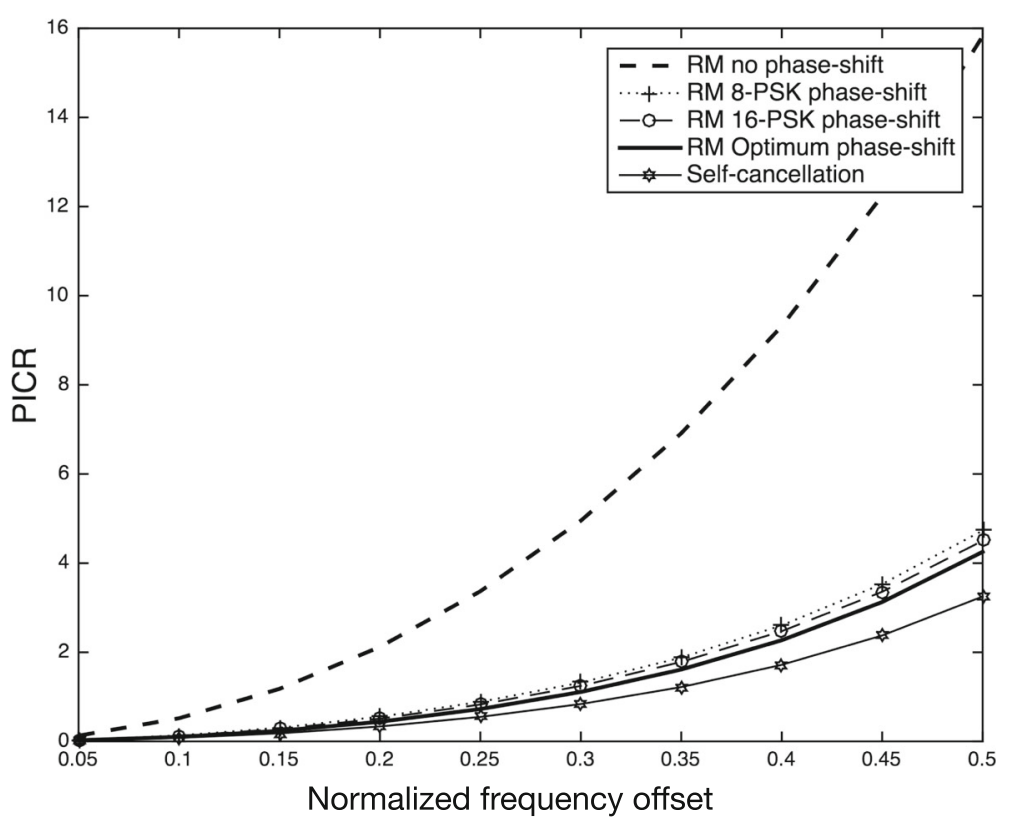

Fig. 3 PICR of $2^{h}$-ary PSK code equivalent to $\operatorname{RM}(1,6)$ 


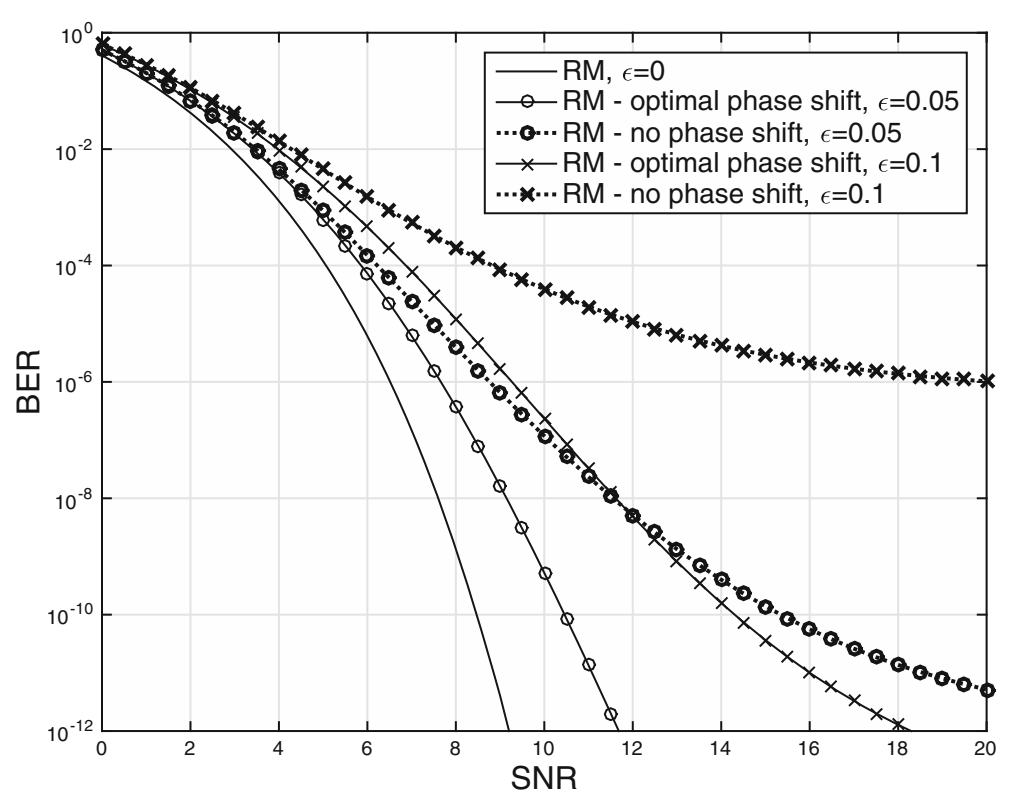

Fig. 4 BER vs. SNR of RM $(1,6)$ code with and without phase-shifts

${ }^{3}$ In the case of non-uniform energy constellation, like QAM, $\mathcal{J}(k, \varepsilon)$ will be determined for each energy level and then minimized over all the levels.

\section{Appendix}

\section{Proof of Theorem 5.1}

The proof follows along the lines of [45]. Based on Eq. (7), we have

$$
\operatorname{PICR}(\mathcal{C}, \varepsilon) \geq \mathcal{J}(0, \varepsilon)
$$

Using the following inequality

$$
\begin{aligned}
x^{2}+y^{2} & =\frac{1}{2}\left[(x+y)^{2}+(x-y)^{2}\right] \\
& \geq \frac{1}{2}(x+y)^{2}, \quad x, y \in R
\end{aligned}
$$

and Eq. (10), we get

$$
\operatorname{PICR}(\mathbf{c}, \varepsilon) \geq \frac{1}{8\left|b_{0}\right|^{2}}\left[2 N+2-2\left|b_{0}\right|^{2}-\left\|\mathbf{c}-\mathbf{w}_{\mathbf{0}}\right\|^{2}-\left\|\mathbf{c}-j \mathbf{w}_{\mathbf{0}}\right\|^{2}\right]^{2}
$$

We need some preliminaries in order to prove Theorem 5.1. If we assume that elements of $\mathcal{C}$ have unit energy, It is straightforward to establish that

$$
\left\|\mathbf{c}-\mathbf{w}_{0}\right\|^{2}=1+\sum_{i=1}^{N-1}\left(1-\left|b_{i}\right|\right)^{2}+4 \sum_{i=1}^{N-1}\left|b_{i}\right| \sin ^{2}\left(\frac{\angle b_{i}-\angle c_{i}}{2}\right)
$$

and

$$
\left\|\mathbf{c}-j \mathbf{w}_{\mathbf{0}}\right\|^{2}=1+\sum_{i=1}^{N-1}\left(1-\left|b_{i}\right|\right)^{2}+4 \sum_{i=1}^{N-1}\left|b_{i}\right| \sin ^{2}\left(\frac{\angle b_{i}-\angle c_{i}}{2}+\frac{\pi}{4}\right)
$$

where $|x|$ and $\angle x$ are the amplitude and the angle of $x$, respectively. Let $\mathbb{Z}_{2^{h}}$ denote the ring of integers modulo $2^{h}$. We define $\bar{c}_{i}$ and $\bar{b}_{i} \in \mathbb{Z}_{2^{h}}$ as: $\frac{2 \pi \bar{c}_{i}}{2^{h}} \simeq \angle c_{i}$ and $\frac{2 \pi \bar{b}_{i}}{2^{h}} \simeq$ $\angle b_{i}$. Note that when $h$ tends to the infinity the phases can be approximated with arbitrary high probability.

Schmidt showed in [45] that

$$
\sin ^{2}\left(w \frac{\pi}{2^{h}}\right) \leq \frac{2^{h}}{4} \sin ^{2}\left(\frac{\pi}{2^{h}}\right) w
$$

for $h>1$ and $w=0,1, \ldots, 2^{h-1}$.

Based on this result, we get

$$
\begin{aligned}
\left\|\mathbf{c}-\mathbf{w}_{\mathbf{0}}\right\|^{2} \leq & 1+\sum_{i=1}^{N-1}\left(1-\left|b_{i}\right|\right)^{2}+2^{h} \sin ^{2}\left(\frac{\pi}{2^{h}}\right) \\
& \sum_{i=1}^{N-1}\left|b_{i}\right| \min \left(\overline{b_{i}}-\overline{c_{i}}, 2^{h}-\overline{b_{i}}+\overline{c_{i}}\right),
\end{aligned}
$$

and

$$
\begin{gathered}
\left\|\mathbf{c}-j \mathbf{w}_{\mathbf{0}}\right\|^{2} \leq 1+\sum_{i=1}^{N-1}\left(1-\left|b_{i}\right|\right)^{2}+2^{h} \sin ^{2}\left(\frac{\pi}{2^{h}}\right) \\
\sum_{i=1}^{N-1}\left|b_{i}\right| \min \left(\overline{b_{i}}-\overline{c_{i}}-2^{h-2}, 2^{h}-\overline{b_{i}}+\overline{c_{i}}+2^{h-2}\right) .
\end{gathered}
$$


We now use the previous results to derive a lower bound of the PICR:

$$
\begin{aligned}
& \operatorname{PICR}(\mathbf{c}, \varepsilon) \geq \frac{1}{8\left|b_{0}\right|^{2}}\left[2 N-2\left|b_{0}\right|^{2}-2 \sum_{i=1}^{N-1}\left(1-\left|b_{i}\right|\right)^{2}\right. \\
& \left.-2^{h}\left|b_{\max }\right| \sin ^{2}\left(\frac{\pi}{2^{h}}\right)\left(w t_{L}(\bar{b}-\bar{c})+w t_{L}\left(\bar{b}-\bar{c}-2^{h-2}\right)\right)\right]^{2}
\end{aligned}
$$

where $b_{\text {max }}=\max _{1 \leq i \leq N-1}\left|b_{i}\right|, \bar{b}=\left[\bar{b}_{0}, \ldots, \bar{b}_{N-1}\right], \bar{c}=$ $\left[\bar{c}_{0}, \ldots, \bar{c}_{N-1}\right]$ and $w t_{L}(\mathbf{x})$ is Lee weight of the vector $\mathbf{x}=$ $\left[x_{0}, x_{1}, \ldots, x_{N-1}\right]$ defined as

$$
w t_{L}(x) \triangleq \sum_{i=0}^{N-1} \min \left\{x_{i}, 2^{h}-x_{i}\right\}
$$

Note that this lower bound is not always tight. Therefore, since all the coefficients $b_{k}$ slowly vary with $k, b_{k+1} \simeq$ $b_{k}$ (if $\left.|\epsilon| \ll 1\right)$, we derived this approximated lower bound

$$
\begin{aligned}
\operatorname{PICR}(\mathbf{c}, \varepsilon) \gtrsim \frac{1}{8\left|b_{0}\right|^{2}}\left[2 N-2\left|b_{0}\right|^{2}-2 \sum_{i=1}^{N-1}\left(1-\left|b_{i}\right|\right)^{2}\right. \\
\left.-2^{h}\left|b_{\mathrm{avg}}\right| \sin ^{2}\left(\frac{\pi}{2^{h}}\right)\left(w t_{L}(\bar{b}-\bar{c})+w t_{L}\left(\bar{b}-\bar{c}-2^{h-2}\right)\right)\right]^{2}
\end{aligned}
$$

where $b_{\text {avg }}=\operatorname{mean}_{1 \leq i \leq N-1}\left|b_{i}\right|$. The resulting values are very good approximations to the PICR lower bound yet analytically tractable. We use Definition 12 and Lemma 13 in [45] to derive

$$
w t_{L}(\bar{b}-\bar{c})+w t_{L}\left(\bar{b}-\bar{c}-2^{h-2}\right)=2^{h-2} N+2^{h-1} \rho(\mathcal{B}),
$$

where $\rho(\mathcal{B})$ is covering radius of the binary code $\mathcal{B} \subseteq$ $\{1,-1\}^{N}$. We replace Eq. (20) in Eqs. (17) and (19) to complete the proof.

\section{Competing interests}

The authors declare that they have no competing interests.

Received: 22 March 2016 Accepted: 7 January 2017

Published online: 28 January 2017

\section{References}

1. T Pollet, MV Bladel, M Moeneclaey, Ber sensitivity of OFDM systems to carrier frequency offset and Wiener phase noise. IEEE Trans. Commun. 43(2/3/4), 191-193 (1995). doi:10.1109/26.380034

2. K Sathananthan, C Tellambura, Probability of error calculation of OFDM systems with frequency offset. IEEE Trans. Commun. 49(11), 1884-1888 (2001). doi:10.1109/26.966051
3. JJ van de Beek, M Sandell, PO Borjesson, Ml estimation of time and frequency offset in OFDM systems. IEEE Trans. Signal Process. 45(7), 1800-1805 (1997). doi:10.1109/78.599949

4. U Tureli, D Kivanc, H Liu, Experimental and analytical studies on a high-resolution OFDM carrier frequency offset estimator. IEEE Trans. Veh. Technol. 50(2), 629-643 (2001). doi:10.1109/25.923074

5. MJF-G Garcia, O Edfors, JM Paez-Borrallo, Frequency offset correction for coherent OFDM in wireless systems. IEEE Trans. Consum. Electron. 47(1), 187-193 (2001). doi:10.1109/30.920438

6. M Luise, M Marselli, R Reggiannini, Low-complexity blind carrier frequency recovery for OFDM signals over frequency-selective radio channels. IEEE Trans. Commun. 50(7), 1182-1188 (2002). doi:10.1109/TCOMM.2002.800819

7. $\mathrm{PH}$ Moose, A technique for orthogonal frequency division multiplexing frequency offset correction. IEEE Trans. Commun. 42(10), 2908-2914 (1994). doi:10.1109/26.328961

8. TM Schmidl, DC Cox, Robust frequency and timing synchronization for OFDM. IEEE Trans. Commun. 45(12), 1613-1621 (1997). doi:10.1109/26.650240

9. M Morelli, U Mengali, An improved frequency offset estimator for OFDM applications. IEEE Commun. Lett. 3(3), 75-77 (1999). doi:10.1109/4234.752907

10. M Morelli, U Mengali, Carrier-frequency estimation for transmissions over selective channels. IEEE Trans. Commun. 48(9), 1580-1589 (2000). doi:10.1109/26.870025

11. J Lei, T-S Ng, A consistent ofdm carrier frequency offset estimator based on distinctively spaced pilot tones. IEEE Trans. Wirel. Commun. 3(2), 588-599 (2004). doi:10.1109/TWC.2004.825350

12. Y Li, H Minn, N Al-Dhahir, AR Calderbank, Pilot designs for consistent frequency-offset estimation in OFDM systems. IEEE Trans. Commun. 55(5), 864-877 (2007). doi:10.1109/TCOMM.2007.896105

13. U Tureli, H Liu, MD Zoltowski, OFDM blind carrier offset estimation: Esprit. IEEE Trans. Commun. 48(9), 1459-1461 (2000). doi:10.1109/26.870011

14. K Sathananathan, C Tellambura, in ISCAS 2001. The 2001 IEEE International Symposium on Circuits and Systems (Cat. No. 01CH37196). Forward error correction codes to reduce intercarrier interference in OFDM, vol. 4, (Sydney, 2001), pp. 566-569

15. J Armstrong, Analysis of new and existing methods of reducing intercarrier interference due to carrier frequency offset in OFDM. IEEE Trans. Commun. 47(3), 365-369 (1999). doi:10.1109/26.752816

16. AF Molisch, M Toeltsch, S Vermani, Iterative methods for cancellation of intercarrier interference in OFDM systems. IEEE Trans. Veh. Technol. 56(4), 2158-2167 (2007). doi:10.1109/TVT.2007.897628

17. P Singh, OP Sahu, in 2015 IEEE International Conference on Computational Intelligence \& Communication Technology. An overview of $\mathrm{ICI}$ self cancellation techniques in OFDM systems, (Ghaziabad, 2015). pp. 299-302

18. Y Zhao, SG Haggman, in Proceedings of Vehicular Technology ConferenceVTC. Sensitivity to Doppler shift and carrier frequency errors in OFDM systems-the consequences and solutions, vol. 3, (Atlanta, 1996), pp. 1564-1568

19. Y Zhao, SG Haggman, Intercarrier interference self-cancellation scheme for OFDM mobile communication systems. IEEE Trans. Commun. 49(7), 1185-1191 (2001). doi:10.1109/26.935159

20. K Sathananthan, RMAP Rajatheva, SB Slimane, Cancellation technique to reduce intercarrier interference in OFDM. Electron. Lett. 36(25), 2078-2079 (2000). doi:10.1049/el:20001456

21. YH Peng, YC Kuo, GR Lee, JH Wen, Performance analysis of a new ICl-self-cancellation-scheme in OFDM systems. IEEE Trans. Consum. Electron. 53(4), 1333-1338 (2007). doi:10.1109/TCE.2007.4429221

22. A Singh, A Sharma, in 2015 International Conference on Advances in Computer Engineering and Applications. ICI cancellation in OFDM by phase rotated data transmission, (Ghaziabad, 2015), pp. 952-956

23. K Sathananthan, CRN Athaudage, B Qiu, in Proceedings. ISCC 2004. Ninth International Symposium on Computers And Communications (IEEE Cat. No. 04TH8769). A novel ICl cancellation scheme to reduce both frequency offset and IQ imbalance effects in OFDM, vol. 2, (Alexandria, 2004), pp. 708-713

24. STang, K Gong, J Song, C Pan, Z Yang, Intercarrier interference cancellation with frequency diversity for OFDM systems. IEEE Trans. Broadcast. 53(1), 132-137 (2007). doi:10.1109/TBC.2006.886835 
25. T Shu-Ming, H Yu-Shun, A novel ICI self-cancellation scheme for OFDM systems. Int. J. Commun. Syst. 24(11), 1496-1505 (2011)

26. MW Wen, X Cheng, X Wei, B Ai, BL Jiao, in 2011 IEEE Global Telecommunications Conference - GLOBECOM. A Novel Effective IC Self-Cancellation Method, (Houston, 2011), pp. 1-5

27. TL Liu, WH Chung, SY Yuan, SY Kuo, ICl self-cancellation with cosine windowing in OFDM transmitters over fast time-varying channels. IEEE Trans. Wirel. Commun. 14(7), 3559-3570 (2015). doi:10.1109/TWC.2015.2408325

28. K Sathananthan, C Tellambura, in IEEE 54th Vehicular Technology Conference. VTC Fall 2001. Proceedings (Cat. No. 01CH37211). New ICI reduction schemes for OFDM system, vol. 2, (Atlantic City, 2001), pp. 834-838

29. K Sathananthan, C Tellambura, Partial transmit sequence arid selected mapping schemes to reduce ICI in OFDM systems. IEEE Commun. Lett. 6(8), 313-315 (2002). doi:10.1109/LCOMM.2002.802067

30. A Seyedi, GJ Saulnier, General ICI self-cancellation scheme for OFDM systems. IEEE Trans. Veh. Technol. 54(1), 198-210 (2005). doi:10.1109/TVT.2004.838849

31. O Real, V Almenar, in 2008 IEEE International Conference on Wireless and Mobile Computing, Networking and Communications. OFDM ICl Self-Cancellation Scheme Based on Five Weights, (Avignon, 2008), pp. 360-364

32. HG Yeh, CC Wang, in IEEE 60th Vehicular Technology Conference, 2004. VTC2004-Fall. New parallel algorithm for mitigating the frequency offset of OFDM systems, vol. 3, (Los Angeles, 2004), pp. 2087-2091

33. HG Yeh, YK Chang, B Hassibi, A scheme for cancelling intercarrier interference using conjugate transmission in multicarrier communication systems. IEEE Trans. Wirel. Commun. 6(1), 3-7 (2007). doi:10.1109/TWC.2007.04541

34. CL Wang, YC Huang, Intercarrier interference cancellation using general phase rotated conjugate transmission for OFDM systems. IEEE Trans. Commun. 58(3), 812-819 (2010). doi:10.1109/TCOMM.2010.03.080288

35. HG Yeh, K Yao, in 2012 IEEE Global Communications Conference (GLOBECOM). A parallel ICl cancellation technique for OFDM systems, (Anaheim, 2012), pp. 3679-3684. doi:10.1109/GLOCOM.2012.6503688

36. M Wen, X Cheng, L Yang, B Jiao, in 2013 IEEE Global Communications Conference (GLOBECOM). Two-path transmission framework for $\mathrm{ICl}$ reduction in OFDM systems, (Atlanta, 2013), pp. 3716-3721

37. Y Zhao, JD Leclercq, SG Haggman, Intercarrier interference compression in OFDM communication systems by using correlative coding. IEEE Commun. Lett. 2(8), 214-216 (1998). doi:10.1109/4234.709435

38. H Zhang, Y Li, Optimum frequency-domain partial response encoding in OFDM system. IEEE Trans. Commun. 51(7), 1064-1068 (2003). doi:10.1109/TCOMM.2003.814203

39. JY Yun, SY Chung, YH Lee, Design of ICl canceling codes for OFDM systems based on capacity maximization. IEEE Signal Process. Lett. 14(3), 169-172 (2007). doi:10.1109/LSP.2006.884035

40. B Smida, in 2011 IEEE Global Telecommunications Conference-GLOBECOM. Reduction of the Peak Interference to Carrier Ratio of OFDM Signals, (Houston, 2011), pp. 1-5. doi:10.1109/GLOCOM.2011.6133563

41. AE Jones, TA Wilkinson, SK Barton, Block coding scheme for reduction of peak to mean envelope power ratio of multicarrier transmission schemes. Electron. Lett. 30(25), 2098-2099 (1994). doi:10.1049/el:19941423

42. AE Jones, TA Wilkinson, in Vehicular Technology Conference, 1996. Mobile Technology for the Human Race., IEEE 46th. Combined coding for error control and increased robustness to system nonlinearities in OFDM, vol. 2 (1996), pp. 904-9082. doi:10.1109/VETEC.1996.501442

43. V Tarokh, $\mathrm{H}$ Jafarkhani, On the computation and reduction of the peak-to-average power ratio in multicarrier communications. IEEE Trans. Commun. 48(1), 37-44 (2000). doi:10.1109/26.818871

44. KG Paterson, V Tarokh, On the existence and construction of good codes with low peak-to-average power ratios. IEEE Trans. Inf. Theory. 46(6), 1974-1987 (2000). doi:10.1109/18.868473

45. KU Schmidt, On the peak-to-mean envelope power ratio of phase-shifted binary codes. IEEE Trans. Commun. 56(11), 1816-1823 (2008). doi:10.1109/TCOMM.2008.060652

46. THelleseth, T Klove, J Mykkeltveit, On the covering radius of binary codes (corresp.) IEEE Trans. Inf. Theory. 24(5), 627-628 (1978). doi:10.1109/TIT.1978.1055928
47. SG Vladuts, AN Skorobogatov, Covering radius for long BCH codes. Probl. Inf. Transm. 25(1), 28-34 (1989)

48. RM Pyndiah, Near-optimum decoding of product codes: block turbo codes. IEEE Trans. Commun. 46(8), 1003-1010 (1998). doi:10.1109/26.705396

49. M Sabbaghian, Y Kwak, B Smida, V Tarokh, Near shannon limit and low peak to average power ratio turbo block coded OFDM. IEEE Trans. Commun. 59(8), 2042-2045 (2011). doi:10.1109/TCOMM.2011.080111.090356

50. E Abbe, A Shpilka, A Wigderson, Reed x2013;muller codes for random erasures and errors. IEEE Trans. Inf. Theory. 61(10), 5229-5252 (2015). doi:10.1109/TIT.2015.2462817

\section{Submit your manuscript to a SpringerOpen ${ }^{\circ}$ journal and benefit from:}

- Convenient online submission

- Rigorous peer review

- Immediate publication on acceptance

- Open access: articles freely available online

- High visibility within the field

- Retaining the copyright to your article

Submit your next manuscript at $>$ springeropen.com 